\title{
Sedimentation upon different carrier liquid in giant electrorheological fluid and its application
}

\section{Yaying Hong and Weijia Wen*}

Department of Physics, Hong Kong University of Science and Technology, Hong Kong, China

Edited by:

Weihua Li, University of Wollongong, Australia

\section{Reviewed by:}

Bo Hou, Soochow University, China

Xianzhou Zhang, G.H. Varley

Engineering, Australia

*Correspondence:

Weijia Wen, Department of Physics and Institute of Nano Science and

Technology, The Hong Kong University of Science and Technology, Clear

Water Bay, Kowloon, Hong Kong,

China

e-mail:phwen@ust.hk
When giant electrorheological (GER) fluid is settled after some time, particles can precipitate out of the oil in a multistep process that involves the formation of larger particles, the aggregation of colloids, and eventual sedimentation. Colloidal stability in GER fluid can influence the GER performance and the fluid flow steadiness. We investigated the sedimentation effect of the GER particles suspended in various carrier liquid. Different from the existing electrorheological (ER) fluids, GER particles consisting of oxalate core with urea coating are found oil synergistic. The sedimentation effect of the particles suspended in oils from the family of synthetic oil and mineral oil were checked by direct observation. The rheological behavior of the GER fluid upon electric field application was also investigated. These experiments showed that stable colloidal suspension and good GER effect can be achieved coherently by favorable particle-oil interaction. The resultant high yield stress and low sedimentation rate achieved due to the instrumental linking of hydrogen bond is showed in the hydrogenated silicone oil carrier liquid. With the anti-sedimentation characteristic upon the new carrier oil, hydrogenated silicone oil-GER fluid, we investigated their GER effect in a modified mono tube damper and the experimental result showed wide controllability range. Our investigations may broaden engineering applications.

Keywords: giant electrorheological, sedimentation, GER damper

\section{INTRODUCTION}

Field responsive fluids, such as electrorheological (ER) fluid and magnetorheological (MR) fluid, are a trend to the new generation in design for product where power density, accuracy, and dynamic performance are the key features. These materials are different from the traditional smart materials, in that they are soft materials (typically dispersions or gels) rather than solids. For every system where it is desirable to control motion and vibration using a fluid with changing viscosity, a smart colloid may be an improvement in functionality and costs. Simplicity and more intelligence in the functionality are key features of this technology. Excellent features like fast response, simple interface between electrical power input and the mechanical power output, and controllability make smart material the next technology of choice for many applications (Stanway, 2004; Shen et al., 2006).

One significant discovery in ER field is the giant electrorheological (GER) effect made by Wen et al. (2003). The GER fluid consists of the nanoparticles of oxalate core with urea coating and silicone oil. Other than the breakthrough of the high yield stresses (100-200 kPa), there are other merits of such GER fluid including fast response time, reasonable sedimentation rate as well as high breakdown voltage, which open wide up for different industrial application such as car suspension and robotics.

Due to long inactivity duration of the particles in the fluid, undesired particle aggregation arises in concentrated GER fluid. As a result, the formation of sediments is facilitated. Hence, the poor re-dispersibility arises as a serious problem facing the technological application of the GER fluid. After years of intensive research, a fundamental correlation of the physic-chemical properties involved in the GER effect has not been adequately developed (Cho et al., 2004; Chen and Wei, 2006; Choi and Jhon, 2009). There have been reports on the ER enhancing sedimentation through the dispersing media, surfactants, agents, and none has systematically investigated the role of the dispersing liquid. This is largely due to the conventional wisdom that the dispersing liquid plays only a passive role in providing a large mismatch between the dielectric constants of solid particles and oil. It is known not to be the case for the recently discovered GER effect whose mechanism is based on the alignment of molecular dipoles through the hydrogen bonding network (Huang et al., 2006; Chen et al., 2010).

The aim of the present work is to investigate the role played by carrier liquid in the stability against particle aggregation and settling, the re-dispersibility of concentrated GER suspension and their GER effect. We systematically studied different types of oils including the synthetic oil and mineral oil influencing its colloidal stability by direct observation. The rheological behavior of the GER fluid upon electric field application was also investigated. The results will allow us to determine the relationship of the particle interaction with the carrier liquid. With the less sedimentation effect upon the tested carrier liquid, we investigated its GER performance in a modified mono tube damper for application usage.

\section{MATERIALS AND METHODS SYNTHESIS OF GER SAMPLES}

Our GER particles, $\mathrm{BaTiO}\left(\mathrm{C}_{2} \mathrm{O}_{4}\right)_{2}+\mathrm{NH}_{2} \mathrm{CONH}_{2}$, were fabricated by the modification of Kudaka method in our previous work 
(Gong et al., 2008). Barium chloride solution and oxalic acid solution were separately dissolved in distilled water at $65^{\circ} \mathrm{C}$. Titanium tetrachloride solution was then slowly added to the prepared oxalic acid solution under ultrasonic. Both the solutions were then mixed immediately in an ultrasonic bath at $65^{\circ} \mathrm{C}$ to prevent hydrolysis. Nanometer sized core-shell particles were formed at this stage. Addition of urea, as an ER promoter, to the mixed solution led to the formation of a white colloid. The solution was drastically cooled to room temperature. The precipitate was washed, filtered, and then vacuum-dried at $100^{\circ} \mathrm{C}$ for $2 \mathrm{~h}$ to remove all traces water. All the chemicals aforementioned including two oil samples (white mineral oil and liquid paraffin) were supplied by Sigma Aldrich Chemical Company. Dimethyl terminated and hydrogenated silicone oils were supplied by Dow Corning. All the oils were dried at $120^{\circ} \mathrm{C}$ for $2 \mathrm{~h}$ before the experiment to avoid moisture. GER particles and each of the different oil samples were homogenized in a high-speed grinding mill for at least an hour. Consideration on longer mixing time is needed especially for the mineral oil samples. Concentration of the sample fluids can be denoted as the amount of oil, in units of milliliter, mixed with each gram of GER particles. Hence 0.5 means $10 \mathrm{~g}$ of GER particles mixed with $5 \mathrm{ml}$ of oil.

\section{GER PARTICLE MEASUREMENT}

JEOL-6700F scanning electron microscopy (SEM) with a target acceleration voltage of $5 \mathrm{kV}$ was used to visualize the morphology of the GER particles. A SEM sample was prepared by dispersing $10 \mathrm{mg}$ particle in $2 \mathrm{ml}$ ethanol by ultrasonication. A drop of the suspension sample was transferred to a wafer for volatilization. The sample on the wafer was then gold-coated to enhance the electrical conductivity for the SEM. The size distribution of the batch samples was confirmed by PAnalytical X-Ray diffractometer (X’pert Pro).

\section{RHEOLOGICAL DATA COLLECTION}

A circular-plate type viscometer (Haake RS1), with an $8 \mathrm{~mm}$ diameter rotating disk and a gap of $1 \mathrm{~mm}$ between the rotor and stator, was used to perform the rheological measurements. The step signals for driving the DC high-voltage source (SPELLMAN SL300) were generated by a functional generator (PM 5315, Philips). Software package RHEOWIN was used to collect experimental data. A $50 \mathrm{~s}$ square voltage pulse was applied to the sample with each measurement repeated at least three times due to their reproducibility and repeatability. Shear stress as a function of time was measured at a very low shear rate $\left(0.1 \mathrm{~s}^{-1}\right)$. Yield point was reached when a stress-time curve changed its slope to be flat after an abrupt increase at the beginning of turning on the field. The yield stress at a given field was taken to be the maximum of the shear stress in the corresponding time span. The measurements were performed $24 \mathrm{~h}$ after dispersion at $24^{\circ} \mathrm{C}$.

\section{SEDIMENTATION DATA COLLECTION}

We have measured the sedimentation ratio of four GER fluid samples prepared with same GER particle concentrations of 0.5. Immediately after homogenization, the GER samples were put in a graduated square tube of constantly equal size under ambient gravity conditions. Over time, the settlement level becomes visible as an increasingly sharp interface between the concentrated phase at the bottom and the diluted phase on the top. The homogeneity was determined as a percentage ratio of visible settlement level over the filling level with equation below.

$$
\text { Sedimentation ratio }=\frac{\text { Opaque phase GER fluid }}{\text { Opaque phase }+ \text { Clear oil phase }}
$$

Re-dispersing measurements were carried out on the samples from the sedimentation tests (settling time of 12 weeks). The remixing was done with a planetary shaker, which homogenizes the sample by circular motions of the glass tube. The remixing behavior was determined by the time needed for full homogenization while the vortex-shaker was set to a constant value.

\section{GER DAMPER DATA COLLECTION}

The modified GER damper, filled with hydrogenated silicone oilGER fluid, was first compressed quasi-steadily with a loading speed of $200 \mathrm{~mm} / \mathrm{min}$ on the UTM SINTECH 10/D. During the experiments, the UTM was first turned on to let the crosshead compress the piston rod of the GER damper, and then the electric field was applied to the GER duct when the displacement was about $10 \mathrm{~mm}$. This procedure can avoid device damage as the static yield stress of the GER fluid is very large to cause jamming of the duct We collect the data right after the mixing of the GER fluid and the assembly of the damper. We then left the damper inactive for 3 months and collect data right after inactivity and re-dispersed phase.

\section{RESULTS}

\section{GER PARTICLE CHARACTERIZATION}

The dried GER particles were analyzed by SEM imaging (Figure 1). The particles fabricated are averagely spherical in shape with a regular diameter of $40 \mathrm{~nm}$. X-ray diffractometer showed that the most frequent radius of the size distribution by volume is $15 \mathrm{~nm}$ and the average radius is $22 \mathrm{~nm}$ with a relative standard deviation of $51.97 \%$. We then confirmed our fabricated GER particles match the description from Wen et al. (2004).

\section{RHEOLOGICAL DATA}

The rheological effect of four types of oil samples from two different sources (Table 1) were measured by Haake RS1. Polysiloxanes from synthetic oil are semi-organic polymers and copolymers containing an inorganic backbone of repeating silicon-oxygen units and organic side chains substituted on the silicon atom along the polymer chain. Mineral oil consisted predominantly of carbon and hydrogen, which is non-polar.

With mixture concentration of 0.5 , two extreme visual appearances such as sol-like and clay-like textures were obtained in the samples. When designing an ER application device, the zero-field viscosity of the mixture is always an important factor to be considered. The amplifying ratio is a constant (mixture viscosity divided by oil viscosity), which represented the GER particle interaction with oil. The highest amplifying ratio was credited to white mineral oil, which was meant to have the most unfavorable particle-oil interaction among the tested samples.

To give a simple physical picture of how the particle-oil interaction can play a crucial role in the GER effect, we measured the relevant yield stress (Figure 2) as a function of applied electric field. 


\section{SEDIMENTATION AND RE-DISPERSING EFFECT}

Figure 3 showed the sedimentation ratio of the GER fluid samples within 3 months. It can be seen that the sedimentation rate for white mineral oil carrier liquid decreased initially, for several weeks, and then approached a stable asymptotic value after that. The rest of the tested samples were yet to be sedimented with significant optimum value in 3 months. The most relevant parameter for industrial application is the re-dispersing behavior, which could be improved significantly with hydrogenated silicone oil carrier liquid. As shown in the plotted result, the expended energy necessary for re-homogenization after 3 months of settlement is $15 \%$ less than the white mineral oil carrier liquid. Practically, the settling behavior of the hydrogenated silicone oil-GER fluid became less critical for industrial application where fast access to the full

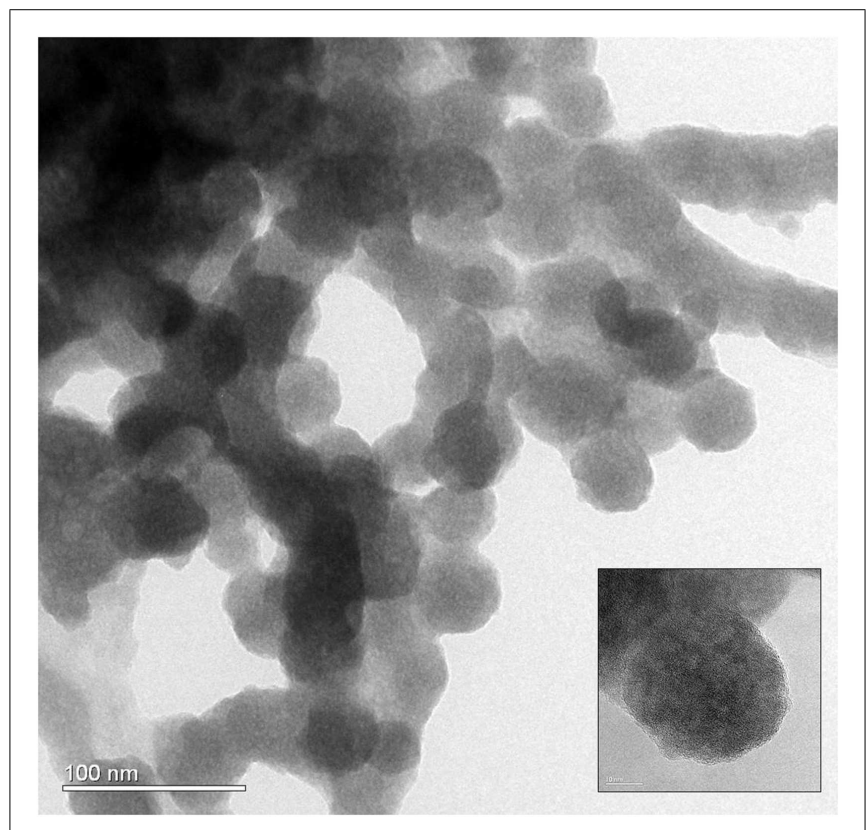

FIGURE 1 | SEM image of the GER particles with an inset of an enlarged GER particle. performance, i.e., damper, is necessary even after a long phase of inactivity.

\section{GER DAMPER TESTING RESULT}

According to Figures 2 and 3, we can see that the best ER effect and sedimentation stability according to their measured characteristic was credited to hydrogenated silicon oil-GER fluid. An GER application test was carried out on a modified commercially available semi-active monotube damper in shear-mode. As shown in Figure 4, we measured the damping force performance of the prototype-test before, after inactivity and re-dispersing phase.

\section{DISCUSSION}

In this study, we investigated the role played by carrier liquid in the stability against particle aggregation and settling, the redispersibility of concentrated GER suspension and their GER effect. Our results show that the hydrogen bondings in the carrier oil enhance the particle-liquid interaction that slowed down sedimentation, enhance easiness of re-dispersibility and maintained good GER performance.

With reference to the results presented in Table 1, some interesting observation found that although hydrogenated silicone oil-GER fluid were having similar mixture viscosity and amplifying ratio with the paraffin oil-GER fluid, the visual appearances are in both extreme. The mixing time needed for the latter samples were two times longer than hydrogenated silicone oil-GER fluid as it appeared that the GER particles in paraffin oil did not spread out evenly. There are many examples of such systems in which non-wetting are a known factor for leading to the same texture as observed in the mineral oil-ER fluid (Conrad and Sprecher, 1991; Shen et al., 2009). Different oil structure can play a significant role in the initial viscosity as well as in the visual appearances of the sample mixtures. The latter is suggestive of a strong interaction between the solid particles and oils.

In order to further connect the experimental results aforementioned, we found two motivating point. First, the results violated the norm for conventional ER fluid that high zero-field viscosity is usually accompanied by high yield stress (Halsey, 1992). Second, the plotted graphs contrary the conventional ER fluids that are only sensitive to the complex dielectric constant of the oil (Ma

Table 1 | Rheological data of various oil-GER fluids.

\begin{tabular}{|c|c|c|c|c|c|c|}
\hline Source & Oil type & Chemical formulae & $\begin{array}{l}\text { Oil viscosity } \\
\text { (m.Pas) }\end{array}$ & $\begin{array}{l}\text { Mixture viscosity } \\
\text { (m.Pas) }^{\mathrm{a}}\end{array}$ & $\begin{array}{l}\text { Visual } \\
\text { appearance }\end{array}$ & $\begin{array}{l}\text { Amplifying } \\
\text { ratio }\end{array}$ \\
\hline \multirow[t]{2}{*}{ Synthetic oil } & Dimethyl terminated silicone oil & & 12 & 169 & Liquid-like & 14.1 \\
\hline & Hydrogenated silicone oil & 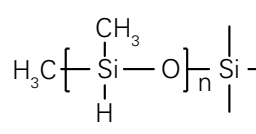 & 20 & 400 & Liquid-like & 20 \\
\hline \multirow[t]{2}{*}{ Mineral oil } & White mineral oil & $\mathrm{C}_{25} \mathrm{H}_{43} \mathrm{NO}_{3}$ & 4 & 359 & Clay-like & 90 \\
\hline & Liquid paraffin & $\mathrm{C}_{n} \mathrm{H}_{2 n+2} n=16 \sim 24$ & 20 & 477 & Clay-like & 23.85 \\
\hline
\end{tabular}

${ }^{a}$ Mixture volume concentration $=0.5$. 


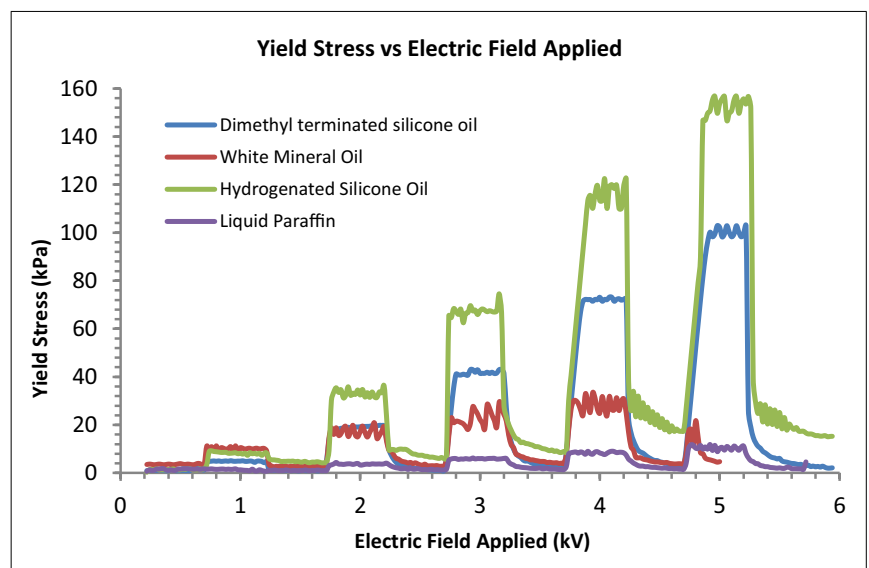

FIGURE 2 |Yield stresses of various oil-GER fluids with volume concentration of $\mathbf{0 . 5}$.

et al., 2003). Figure 2 proofed that GER fluids are oil-sensitive upon their GER effect. For example, with hydrogenated silicone oil, one can obtain a very significant GER effect, but with the same particles dispersed in liquid paraffin, the GER effect is trivial.

Sedimentation occurs in colloidal systems due to the density mismatch between the solid and fluid phases, accentuated by particles aggregation through van der Walls interaction between the particles and the non-favorable particle-solvent interactions. Figure 3 presented the sedimentation rate of the GER particles in various oils. Summarizing the plotted results, the particle-oil interaction contributes massive impact in the sedimentation effect, i.e., bad particle-oil interaction in white mineral oil and good particle-oil interaction in hydrogenated silicone oil. For the bad interaction case, GER particles are phase separated from the oil and the aggregation between two solid are large even with electric field applied. Hence, there can be no yield stress or even induce arcing since the solid aggregates and are always separated by oil (mineral oil family). Arcing is not allowed in the GER fluid system since they will cause irreversible destroy to the fluid. This phenomenon is shown (Figure 2) at the applied electric field of $5 \mathrm{kV} / \mathrm{mm}$ with white mineral oil-GER fluid.

The anti-sedimentation characteristic exhibited by the hydrogenated silicone oil-GER fluid is attributable to the fact that the oil has a very favorable particle-solvent interaction. Figure 5A illustrated the hydrogen bond effectively prevented direct contact between the GER particles, thus minimizing their aggregation and sedimentation. The hydrogen bond acted as the dispersant by suppressing colloidal aggregation in the GER fluid, and therefore also slowed down the separation from the bulk, as revealed through macroscopic sedimentation experiment.

On the other hand for the explanation of GER effect, the surface tension between the GER particles and hydrogenated silicone oil-ER fluid is greatly reduced due to the mediating effect of the hydrogen atom, thus allowing the particles to disperse and to move close together upon the application of an electric field. High GER effect is attributed to the presence of oxalate groups in the core nanoparticles and to the non-uniformity of the urea coating with the modified hydrogen atom in the oil (Figure 5B). The formation

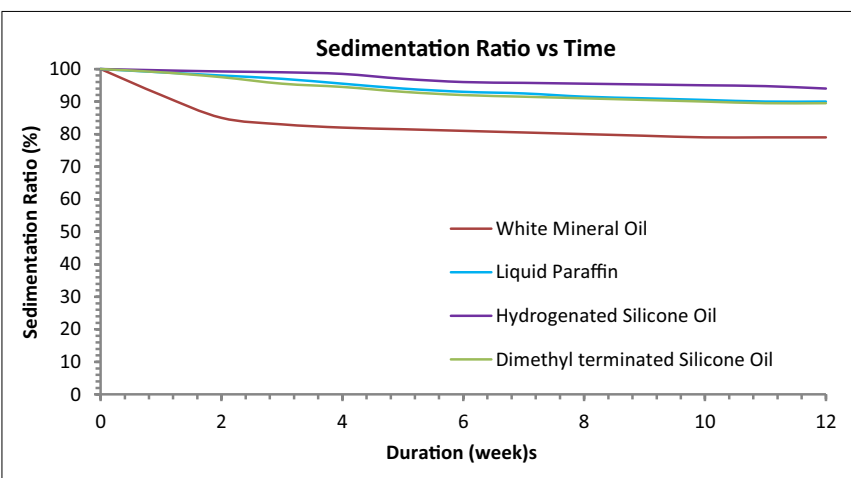

FIGURE 3 | Sedimentation effects of various oil-GER fluids with volume concentration of 0.5 .
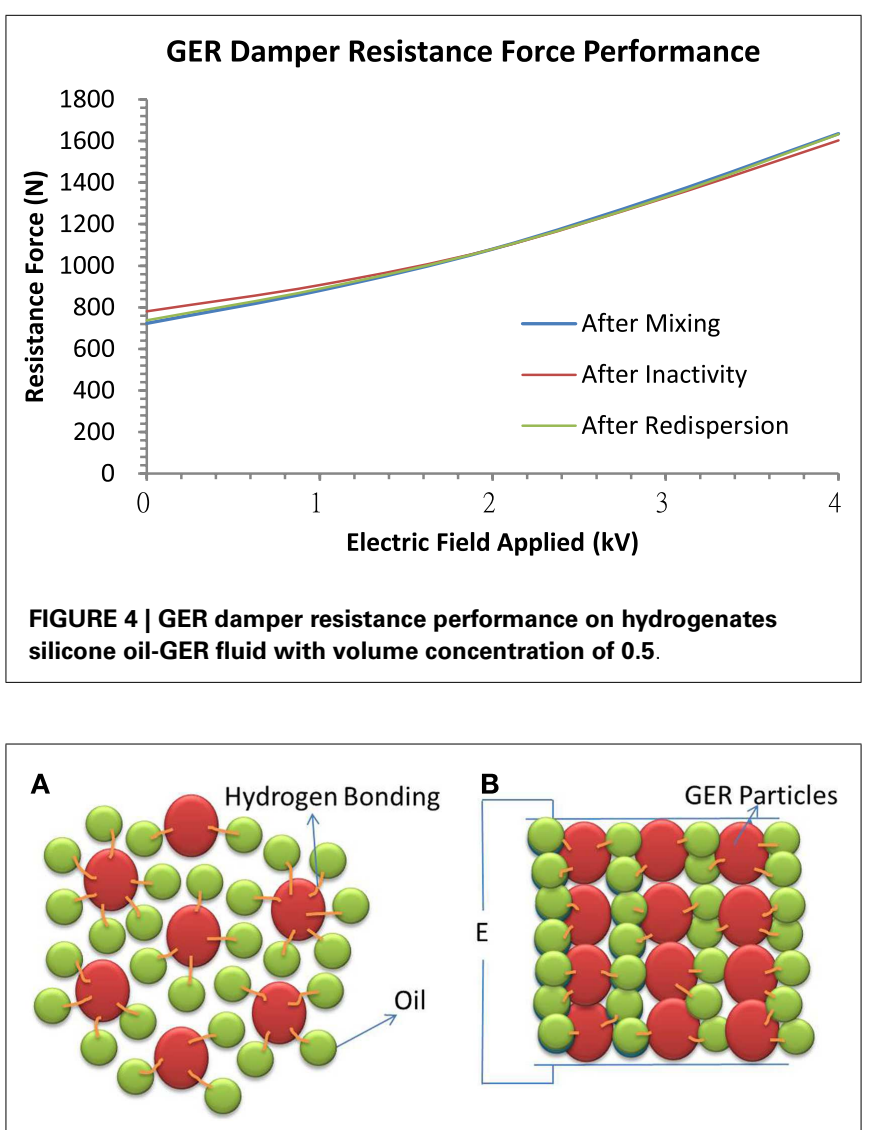

FIGURE 5 | Good particle-solvent interaction of GER fluid (A) without electric field (B) with electric field.

of the filaments, with an attendant lowering of the aligning field and a finite penetration length, can be attributed to the confinement effect exerted by oil chains. Urea molecules have a strong tendency to form hydrogen bonds with one another, and this influences their interactions with hydrophobic oil chains, which are incapable of forming hydrogen bonds.

Figure 4 showed the quasi-steady loading curves of the GER damper under different electric field intensities. It can be seen that 
even when the electric field intensity is zero $(\mathrm{E}=0 \mathrm{kV} / \mathrm{mm})$, there is still a resistant force due to the gas chamber loaded with high pressure nitrogen gas. By increasing the electric field, the resistance force increased. However, the fluctuation of the force is very obvious, because the real flow inside the ER duct is not continuous. It is revealed in the result that sedimentation rate of the hydrogenated silicone oil-ER fluid works reasonably acceptable in GER damper with respect to their needed performance. Dynamic measurement was not performed as the damping force is still difficult to analyze. After some long duration test, the fluid and the sealing of the damper were affecting the output data due to the abrasiveness of the GER particles.

In summary, our study shows that the carrier liquid has synergistic effect on the GER particles. We demonstrated that good particle-oil interaction results in low sedimentation rate and high GER effect with good re-dispersibility after long inactivity duration. Our limitation of study is that our experiments have thus far been conducted only on quasi-steady loading GER damper performance. We did not construct well on the dynamic behavior of the GER fluid flow in the damper. Thus, we cannot discount the possibility of the colloidal stability enhancement during the dynamic fluid flow.

\section{ACKNOWLEDGMENTS}

The authors acknowledge the financial support provided by the Hong Kong NSFC/RGC joint grant of N_HKUST601/11.

\section{REFERENCES}

Chen, S., Huang, X., van der Vegt, N. F. A., Wen, W., and Sheng, P. (2010). Giant electrorheological effect: a microscopic mechanism. Phys. Rev. Lett. 105, 046001. doi:10.1103/PhysRevLett.105.046001

Chen, S., and Wei, C. (2006). Experimental study of the rheological behavior of electrorheological fluids. Smart Mater. Struct. 15, 377. doi:10.1088/0964-1726/ $15 / 2 / 018$

Cho, M., Choi, H., and Ahn, W. (2004). Enhanced electrorheology of conducting polyaniline confined in MCM-41 channels. Langmuir 20, 202-207. doi:10.1021/la035051z

Choi, H., and Jhon, M. (2009). Electrorheology of polymers and nanocomposites. Soft Matter 5, 1562-1567. doi:10.1039/b818368f
Conrad, H., and Sprecher, A. (1991). Characteristic and mechanisms of electrorhreological fluids. J. Stat. Phys. 64, 1073-1091. doi:10.1007/BF01048815

Gong, X., Wu, J., Huang, X., Wen, W., and Sheng, P. (2008). Influence of liquid phase on nanoparticle-based giant electrorheological fluid. Nanotechnology 19, 165602. doi:10.1088/0957-4484/19/16/165602

Halsey, T. (1992). Electrorheological Fluids. Science 258, 761-766. doi:10.1126/ science.258.5083.761

Huang, X., Wen, W., Yang, S., and Sheng, P. (2006). Mechanisms of the giant electrorheological effect. Solid State Commun. 139, 581-588. doi:10.1016/j.ssc.2006. 04.042

Ma, H., Wen, W., Tam, W. Y., and Sheng, P. (2003). Dielectric electrorheological fluids: theory and experiments. Adv. Phys. 343-383. doi:10.1080/ 0001873021000059987

Shen, C., Wen, W., Yang, S., and Sheng, P. (2006). Wetting-induced electrorheological effect. J. Appl. Phys. 99, 106104. doi:10.1063/1.2199749

Shen, R., Wang, X., Lu, Y., Wang, D., and Sun, G. (2009). Polar molecule dominated electrorheological fluids featuring high yield stresses. Adv. Mat. 22, 4631-4635. doi:10.1088/0953-8984/22/32/324105

Stanway, R. (2004). Smart fluid: current and future developments. Mat. Sci. Technol. 20, 931-939. doi:10.1179/026708304225019867

Wen, W., Huang, X., and Sheng, P. (2004). Particle size scaling of the giant electrorheological effect. Appl. Phys. Lett. 85, 299-301. doi:10.1063/1.1772859

Wen, W., Huang, X., Yang, S., Lu, K., \& Sheng, P. (2003). The giant electrorheological effect in suspensions of nanoparticles. Nat. Mater. 2, 727-730. doi:10.1038/nmat993

Conflict of Interest Statement: The authors declare that the research was conducted in the absence of any commercial or financial relationships that could be construed as a potential conflict of interest.

Received: 26 September 2014; accepted: 08 October 2014; published online: 28 October 2014.

Citation: Hong $Y$ and Wen $W$ (2014) Sedimentation upon different carrier liquid in giant electrorheological fluid and its application. Front. Mater. 1:24. doi: 10.3389/fmats.2014.00024

This article was submitted to Smart Materials, a section of the journal Frontiers in Materials.

Copyright $\odot 2014$ Hong and Wen. This is an open-access article distributed under the terms of the Creative Commons Attribution License (CC BY). The use, distribution or reproduction in other forums is permitted, provided the original author(s) or licensor are credited and that the original publication in this journal is cited, in accordance with accepted academic practice. No use, distribution or reproduction is permitted which does not comply with these terms. 